\title{
Oxidized Low-Density Lipoprotein Promotes Macrophage Lipid Accumulation via the Toll-Like Receptor 4-Src Pathway
}

\author{
Ke Yang, PhD; Xiaoqun Wang, MD, PhD; Zhuhui Liu, MD, PhD; Lin Lu, MD, PhD; \\ Jinyan Mao, BSc; Hua Meng, BSc; Yanan Wang, BSc; Yong Hu, MD, PhD; Ying Zeng, MD, PhD; \\ Xiaojie Zhang, BSc; Qiujing Chen, BSc; Yan Liu, MD, PhD; Weifeng Shen, MD, PhD
}

\begin{abstract}
Background: Uptake of oxidized low-density lipoprotein (oxLDL) by macrophages is recognized as a crucial step in the development of atherosclerosis, whereas the precise molecular mechanisms involving it remain to be elucidated.
\end{abstract}

\begin{abstract}
Methods and Results: This study focused on determining the role of toll-like receptor 4 (TLR4) and Src kinase in macrophage lipid accumulation. oxLDL significantly enhanced Src kinase activity and intracellular lipid contents in RAW264.7 macrophages, whereas the small interference RNA-mediated knockdown of TLR4 and Src or chemical inhibition of Src activity blocked oxLDL-induced lipid accumulation. Immunoprecipitation and immunofluorescence studies demonstrated that TLR4 was associated with Src on the plasma membrane upon oxLDL stimulation.
\end{abstract}

Conclusions: The results of the present study suggest an essential role of TLR4-Src signaling in macrophages in the pathogenesis of atherosclerosis. (Circ $J$ 2015; 79: 2509-2516)

Key Words: Atherosclerosis; Lipid; Macrophage; Src; Toll-like receptor 4 (TLR4)

$\mathbf{R}$ etention of lipoprotein in macrophages constitutes a major cause for coronary artery disease by initiating and enhancing local inflammation. ${ }^{1-3}$ Deposition of oxidized low-density lipoprotein (oxLDL) within the subendothelial space of arterial wall recruits circulating monocytes into the intima. ${ }^{4}$ Monocyte-derived macrophages absorb excessive lipids and secrete an array of pro-inflammatory cytokines that accelerate the progression of atherosclerosis. ${ }^{5-7}$ A variety of lipoprotein receptors, including CD36 and lectinlike oxidized LDL receptor-1 (LOX-1), have been identified. ${ }^{8,9}$ Previously, we and others have demonstrated that macrophage lipid accumulation is also regulated by toll-like receptor 4 (TLR4). ${ }^{10-12}$

\section{Editorial $\mathrm{p} 2332$}

TLR4 was first identified to play a fundamental role in innate immunity by detecting lipopolysaccharide (LPS) and activating downstream signals, including Src kinase. As a non-recep- tor tyrosine kinase, $\mathrm{Src}$ is the prototype of a family of highly conserved proteins implicated in diverse signaling pathways and cellular processes. ${ }^{13}$ Src may function as an 'integrator' of multiple endogenous and exogenous stimuli to control cell growth, ${ }^{14,15}$ migration, ${ }^{16,17}$ adhesion ${ }^{18,19}$ and lipid metabolism ${ }^{20,21}$ in macrophages.

In this study, we hypothesized that the TLR4-Src pathway is essential for oxLDL-induced lipid uptake in macrophages. Using immunohistochemistry, we show that phosphorylated Src and TLR4 are increased in macrophages in atherosclerotic plaques. We further demonstrate that Src phosphorylation at tyrosine 418 by TLR4 is involved in macrophage lipid accumulation. These results provide a novel pathway that may advance the understanding of coronary artery disease.

Reagents and Antibodies

Oxidized LDL (Serotec, Oxford, UK) was used to stimulate

Received March 25, 2015; revised manuscript received July 31, 2015; accepted August 17, 2015; released online September 24,2015 Time for primary review: 36 days

Institute of Cardiovascular Disease, Ruijin Hospital, Jiaotong University School of Medicine, Shanghai (K.Y., X.W., Z.L., L.L., J.M., H.M., Y.W., X.Z., Y.L., W.S.); Department of Cardiology, Ruijin Hospital, Jiaotong University School of Medicine, Shanghai (K.Y., X.W., Z.L., L.L., J.M., X.Z., Q.C., Y.L., W.S.); and Department of Regenerative Medicine, Tongji University School of Medicine, Shanghai (Y.H., Y.Z.), China

The first three authors contributed equally to this study (K.Y., X.W., Z.L.).

Mailing address: Weifeng Shen, MD, PhD, Institute of Cardiovascular Disease, Jiaotong University School of Medicine, Ruijin Hospital, 197 Rui Jin Road II, Shanghai 200025, People's Republic of China. E-mail: rjshenweifeng@gmail.com or Yan Liu, MD, PhD, Department of Cardiology, Ruijin Hospital, 197 Rui Jin Road II, Shanghai 200025, People’s Republic of China. E-mail: liuyan_ivy@126.com

ISSN-1346-9843 doi:10.1253/circj.CJ-15-0345

All rights are reserved to the Japanese Circulation Society. For permissions, please e-mail: cj@j-circ.or.jp 
A
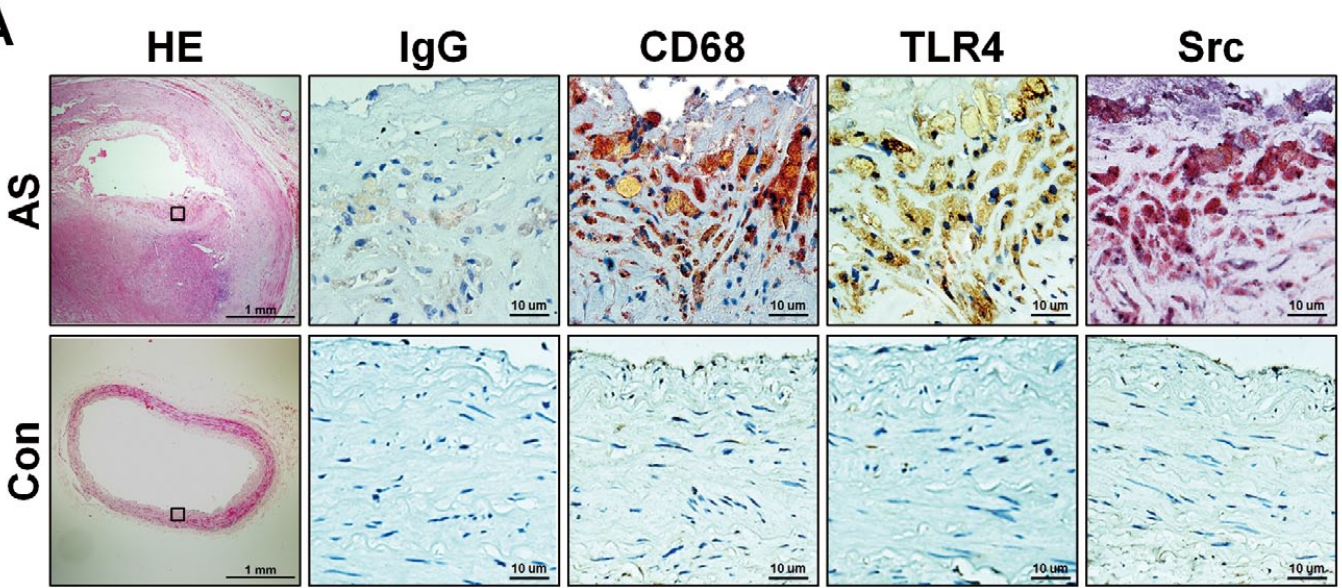

B
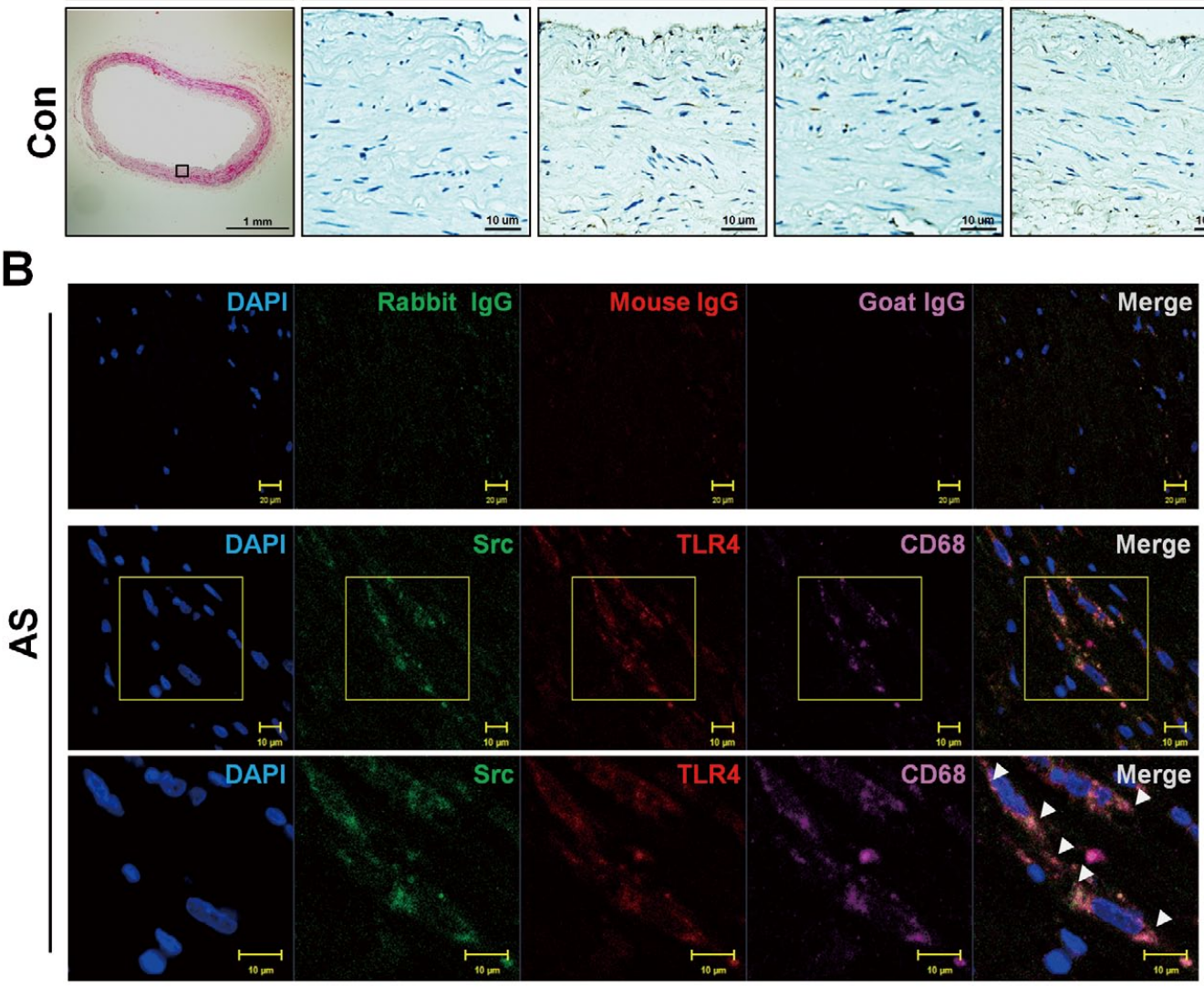

Figure 1. Expression of toll-like receptor 4 (TLR4) and Src in atherosclerotic lesions. (A) Human femoral arteries with atherosclerotic lesions and normal internal thoracic arteries were examined by immunohistochemical analysis. Paraffin-embedded sections were stained with hematoxylin and eosin, anti-CD68, anti-TLR4, anti-p-Src, and control immunoglobulin G (IgG) as negative control. (B) Immunofluorescence staining of the artery with 4',6-diamidino-2-phenylindole (DAPI) (blue), rabbit anti-Src or rabbit IgG (green), mouse anti-TLR4 or mouse IgG (red), goat anti-CD68 or goat IgG (pink), respectively. A gray color in the merge indicates co-localization. Results are representative of 3 independent experiments. AS, atherosclerosis; Con, control.

RAW264.7 cells. LDL was purified to homogeneity by ultracentrifugation, oxidized using $20 \mu \mathrm{mol} / \mathrm{L}$ cupric sulphate in phosphate buffered saline (PBS) at $37^{\circ} \mathrm{C}$ for $24 \mathrm{~h}$ before oxidation was terminated with excess Ethylene Diamine Tetraacetic Acid (EDTA). The level of oxidation was measured using thiobarbituric acid reactive (TBAR) determination, using a malondialdehyde standard. The migration factor of the oxidized LDL was 2.2. Specific small interference RNAs (siRNA) were used for the knockdown of TLR4 and Src (SMART pool TM; Dharmacon, CO, USA). Phorbol 12-myristate 13-acetate, 4-Amino-5-(4-chlorophenyl)-7-(t-butyl) pyrazolo-(3, 4-d) pyrimidine (PP2) and 4-Amino-7-phenylpyrazol (3, 4-d) pyrimidine (PP3, a negative control for PP2) were obtained from CalBiochem (CA, USA). L-glutamine, Oil red O (ORO),
Triton X 100, Hematoxylin, AEC Staining Kits, Neutral red, 3, 3'-Diaminobenzidine, and the BCIP ${ }^{\circledR} /$ NBT Liquid Substrate System were purchased from Sigma-Aldrich (MO, USA). The primary antibodies used included: $\beta$-actin, tumor necrosis factor $\alpha$-associated factor (TRAF) 6, Fyn, Lyn, CD36 and LOX-1 (Cell Signaling, MA, USA), p-Src Tyr418, Src (Abcam, MA, USA), and TLR4 (Invitrogen, CA, USA). HRP-, AP-, Alexa 549-, Alexa 647- and Alexa 488-conjugated antibodies (Cell Signaling) were used as secondary antibodies. The immunohistochemical antibodies used included CD68, TLR4 and Src (Abcam).

\section{Clinical Samples}

Human femoral arteries with atherosclerotic plaques were 
obtained from 3 patients undergoing leg amputation. The internal thoracic arteries $(n=3)$ were obtained and used as normal arteries. The study protocol was approved by the Ethics Committee of Rui Jin Hospital, Shanghai Jiaotong University School of Medicine, and written informed consent was obtained from all patients and donors.

\section{Cell Culture}

Primary human monocytes were isolated from buffy coats derived from healthy donors. Cells were maintained in Roswell Park Memorial Institute medium (RPMI) supplemented with $20 \%$ heat-inactivated fetal calf serum, $1 \%$ penicillin/streptomycin, and $2 \%$ L-glutamine for 5 days. RAW264.7 cells (ATCC, VA, USA) were seeded in 6-well plates at a density of $1.0 \times 10^{6}$ cells per well and cultured in Dulbecco's modified Eagle's medium (DMEM) containing 10\% fetal bovine serum (FBS).

\section{Oligonucleotide and Plasmid Transfection}

Transient transfection of RAW264.7 cells with a negative control, TLR4-, Src- or TRAF6-specific siRNA (100 nmol/L per $10^{5}$ cells) was conducted by using Lipofectamine reagent (Invitrogen).

\section{Assessment of Intracellular Lipids and Total Cholesterol}

After oxLDL stimulation, cultured macrophages in 6-well plates were washed with PBS (Gibco, NY, USA) and then fixed in $4 \%$ paraformaldehyde/PBS for $15 \mathrm{~min}$. After rinsing with $\mathrm{ddH}_{2} \mathrm{O}$, macrophages were stained with $0.3 \%$ Oil red $\mathrm{O}$ (ORO) in 60\% isopropanol for $10 \mathrm{~min}$. After rinsing with PBS for another 4 times, cells were photographed under a microscope (Olympus Microsystems) at $40 \times$ magnification. Five randomly selected fields of 3 independent experiments were analyzed and showed typical images in each group. Quantification of the ORO staining was then performed by elutingstained oil droplets in $100 \%$ isopropanol for $10 \mathrm{~min}$. Optical density was then measured at $500 \mathrm{~nm}$ wavelengths and equalized with the cell numbers analyzed by microscope. Quantifications of total cholesterol were performed following the protocols from the manufacturer (Biovision, CA, USA). Cholesteryl ester was hydrolyzed into free cholesterol by using cholesterol esterase. Total cholesterol was then detected by using a colorimetric method and expressed as relative values compared to total protein concentration $(n=3) .{ }^{22}$

\section{Immunohistochemistry}

Human femoral arteries $(n=3)$ and internal thoracic arteries $(n=3)$ were used for histological and immunochemical analysis. Samples were fixed in $4 \%$ paraformaldehyde overnight, embedded with an optimal cutting temperature (OCT) compound, and cut into serial cryosections ( $6 \mu \mathrm{m}$ thickness). Sections were used for hematoxylin and eosin staining or immunohistochemistry analysis with the following antibodies: anti-CD68 (1:50), anti-TLR4 (1:50) or anti-Src (1:50). After incubation with horseradish peroxidase (HRP) or alkaline phosphatase (AP)-conjugated secondary antibodies (1:100), sections were incubated with 3-amino-9-ethylcarbazole (AEC), 3, 3'-Diaminobenzidine, or 5-Bromo-4-chloro-3-indolyl phosphate/Nitro blue tetrazolium (BCIP/NBT) and counterstained with hematoxylin or Neutral red.

For immunofluorescence analysis, samples were immunostained with anti-TLR4 (1:50) and anti-CD68 (1:50) antibodies for $12 \mathrm{~h}$ at $4^{\circ} \mathrm{C}$ and incubated with Alex (for TLR4) 549- and (for CD68) 647-conjugated secondary antibody $(1: 1,000)$. The sections were then incubated with an anti-Src antibody (1:50) for $12 \mathrm{~h}$ at $4^{\circ} \mathrm{C}$ and then incubated with Alex 488-conjugated

\begin{tabular}{|c|c|}
\hline & Patients $(n=3)$ \\
\hline Male gender $(n, \%)$ & $3(100)$ \\
\hline Age (years) & $59 \pm 1$ \\
\hline Cigarette smoking (n, \%) & $2(66.7)$ \\
\hline Hypertension (n, \%) & $2(66.7)$ \\
\hline Systolic BP (mmHg) & $146 \pm 21$ \\
\hline Diastolic BP (mmHg) & $87 \pm 15$ \\
\hline Type 2 diabetes (n, \%) & 1 (33.3) \\
\hline BMI $\left(\mathrm{kg} / \mathrm{m}^{2}\right)$ & $29.0 \pm 0.6$ \\
\hline Total cholesterol $(\mathrm{mmol} / \mathrm{L})$ & $4.76 \pm 1.43$ \\
\hline HDL-cholesterol (mmol/L) & $1.05 \pm 0.33$ \\
\hline LDL-cholesterol (mmol/L) & $3.62 \pm 0.77$ \\
\hline Triglycerides $(\mathrm{mmol} / \mathrm{L})$ & $1.09 \pm 0.60$ \\
\hline Lipoprotein (a) (g/L) & $0.31 \pm 0.08$ \\
\hline Apolipoprotein (a) (g/L) & $1.23 \pm 0.09$ \\
\hline Fasting glucose (mmol/L) & $4.83 \pm 2.06$ \\
\hline 2-h postprandial glucose $(\mathrm{mmol} / \mathrm{L})$ & $8.83 \pm 4.29$ \\
\hline $\mathrm{HbA} 1 \mathrm{c}(\%)$ & $6.63 \pm 1.18$ \\
\hline Blood urea nitrogen $(\mathrm{mmol} / \mathrm{L})$ & $5.13 \pm 0.80$ \\
\hline Creatinine $(\mu \mathrm{mol} / \mathrm{L})$ & $81.33 \pm 17.50$ \\
\hline Uric acid $(\mu \mathrm{mol} / \mathrm{L})$ & $237.33 \pm 35.44$ \\
\hline
\end{tabular}

BMI, body mass index; BP, blood pressure; HbA1c, hemoglobin A1c; HDL, high-density lipoprotein; LDL, low-density lipoprotein.

secondary antibody (1:1000). All sections were observed and photographed microscopically (Olympus Microsystems).

\section{Immunoprecipitation and Immunoblotting}

Cell pellets were suspended in Tris-buffer containing 1\% (vol/ vol) Triton X-100 and $0.5 \%$ (wt/vol) sodium dodecyl sulfate (SDS) at $4^{\circ} \mathrm{C}$. After centrifugation at $10,000 \mathrm{~g}$ for $2 \mathrm{~min}$, the cell extracts $(250 \mu \mathrm{g}$ protein) in a final volume of $500 \mu \mathrm{l}$ were precleared for $30 \mathrm{~min}$ with $1.0 \mu \mathrm{g}$ of non-immune IgG. Twenty microliters of a $50 \%$ slurry of Protein A/G sepharose beads (Invitrogen) were added, and after $30 \mathrm{~min}$ of incubation, the solution was centrifuged as above. Supernatants were incubated for $2 \mathrm{~h}$ with $20 \mu \mathrm{l}$ of antibody against human TLR4 (Abcam) or Src at $4{ }^{\circ} \mathrm{C}$, after which $50 \mu \mathrm{l}$ of a $50 \%$ slurry of $\mathrm{A} / \mathrm{G}$ beads were added, and the solution was incubated for an additional hour. After centrifugation as described above, $100 \mu \mathrm{l}$ of the supernatant was added to $50 \mu \mathrm{l}$ of $2 \times$ Laemmli sample buffer $(2.3 \%$ SDS, $10 \%$ glycerol, $100 \mathrm{mmol} / \mathrm{L}$ dithiothreitol, and $0.37 \mathrm{~mol} / \mathrm{L}$ Tris. $\mathrm{HCl}, \mathrm{pH}$ 6.8: final concentration). The pellet was washed 3 times with $1 \mathrm{ml}$ of wash buffer $(50 \mathrm{mmol} / \mathrm{L}$ HEPES, $\mathrm{pH} 7.4$, containing $1 \%$ Triton $\mathrm{X}-100,0.1 \% \mathrm{SDS}, 150 \mathrm{mmol} / \mathrm{L} \mathrm{NaCl}$, $100 \mathrm{mmol} / \mathrm{L} \mathrm{NaF}$, and $2 \mathrm{mmol} / \mathrm{L}$ sodium orthovanadate) $\mathrm{Mem}-$ branes were blocked with 5\% BSA in TBS-Tween 20 (TBST). Immunoblots were then performed using antibodies diluted in 1\% BSA TBST. Antibodies against TLR4 (1:500 dilution), Src (1:1,000 dilution), Fyn (1:1,000 dilution) and Lyn (1:1,000 dilution) were used. HRP-conjugated secondary antibodies (Cell signaling) were used to visualize immunoblots. Images were captured and the density of each band was analyzed with GelDoc software (Bio-Rad, Munich, Germany).

\section{Pretreatment With PP2 and PP3}

RAW264.7 cells were treated with PP2 $(10 \mu \mathrm{mol} / \mathrm{L})$ or PP3 $(10 \mu \mathrm{mol} / \mathrm{L})$ for $30 \mathrm{~min}$ prior to the addition of oxLDL. PP3 served as negative control for PP2. 

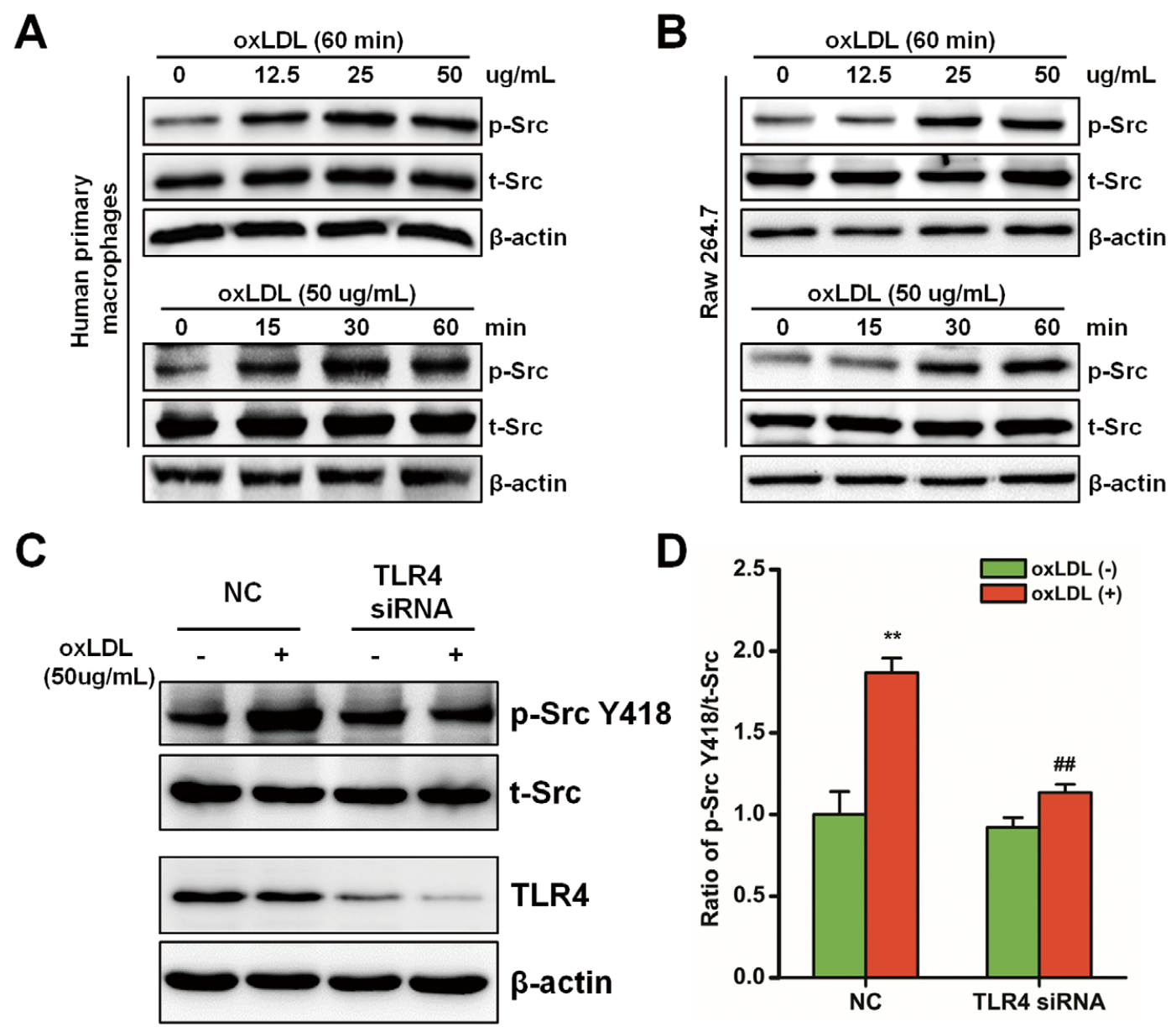

Figure 2. Oxidized low-density lipoprotein (oxLDL) induces toll-like receptor 4 (TLR4)-dependent Src phosphorylation in macrophages. Human primary macrophages and RAW264.7 cells were incubated with oxLDL for indicated times at varying doses. Phosphorylation of Src was detected by Western blotting (A,B). (C) RAW264.7 cells transfected with a negative control or TLR4specific small interference RNAs (siRNA) were incubation with or without oxLDL. Src phosphorylation and TLR4 expression were detected by Western blotting. (D) Densitometric quantifications are shown. Data are expressed as mean \pm SEM from 3 independent tests. ${ }^{* *} \mathrm{P}<0.01$ when compared with oxLDL (-) cells; ${ }^{\# \#} \mathrm{P}<0.01$ when compared with a negative control (NC).

\section{Statistical Analysis}

All values are expressed as mean $\pm S D$. A Student's paired t-test was performed for comparison of paired samples, and ANOVA was used for multiple group comparisons, followed by Friedman's post-test. A probability $(\mathrm{P})$ value $<0.05$ was considered significant.

\section{Results}

\section{TLR4 and Src Co-Localize in Macrophages in Atherosclerotic Lesions}

To investigate the expression and cellular localization of TLR4 and Src in atherosclerotic lesions, immunohistochemistry was performed in human femoral arteries containing atherosclerotic lesions from patients undergoing leg amputation (Figure 1A, top 5 panels; baseline characteristics in Table). Consecutive serial sections showed macrophages (CD68-positive cells) that enriched around the lipid core of atheroma expressed high levels of TLR4 as well as Src, in contrast to the negative staining for TLR4 and Src in normal internal thoracic arteries (Figure 1A, bottom 5 panels). Moreover, co-localization of TLR4, Src and CD68 in the atherosclerotic lesions was confirmed by immunofluorescence staining (Figure 1B).

\section{TLR4 Signaling Regulates Src Kinase Activity Upon oxLDL Stimulation}

Human primary macrophages and RAW264.7 cells were treated with different doses or varying time intervals of oxLDL. We found that oxLDL promoted phosphorylation of Src kinase at Y-418 in a time- and dose-dependent manner, which peaked at $50 \mu \mathrm{g} / \mathrm{ml}$ after incubation for $60 \mathrm{~min}$ (Figures $2 \mathrm{~A}, \mathbf{B}$ ). In addition, boiled oxLDL no longer increased Src phosphorylation, excluding the possibility of LPS contamination (Figure S1). Next, TLR4-specific and negative control (NC) siRNA were transfected into RAW264.7 cells. Src kinase phosphorylation caused by oxLDL was significantly attenuated after TLR4 knockdown (Figures 2C,D), demonstrating that TLR4 signaling mediates Src kinase activation in response to oxLDL exposure. 
A

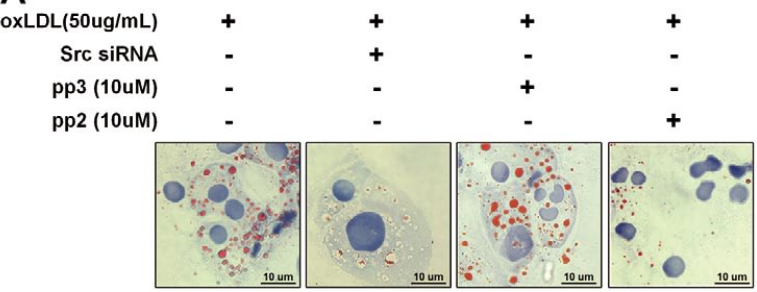

B

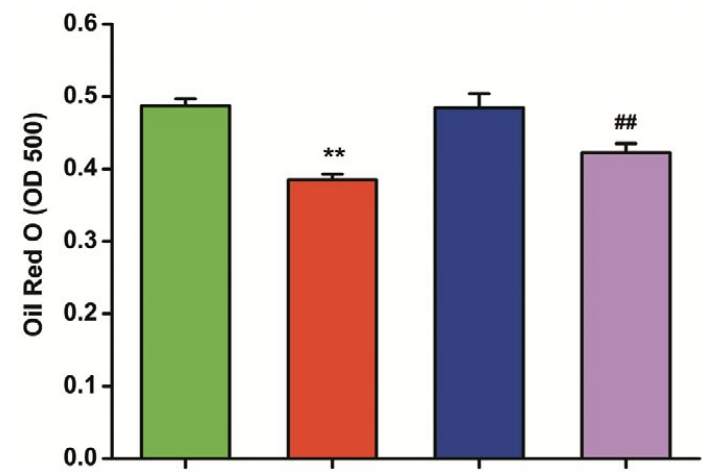

C

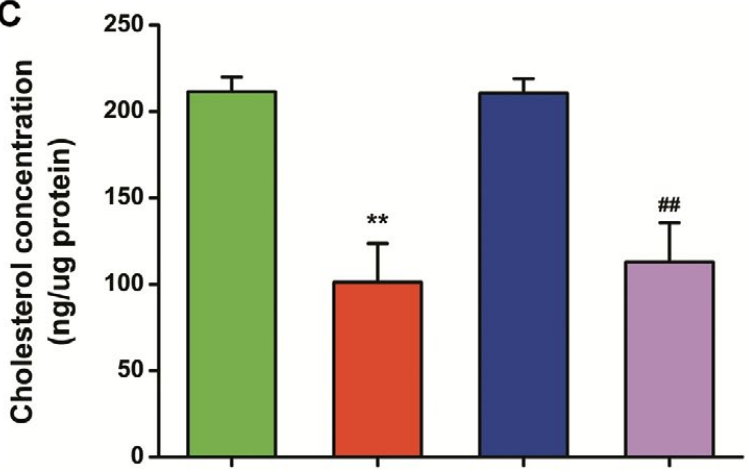

D

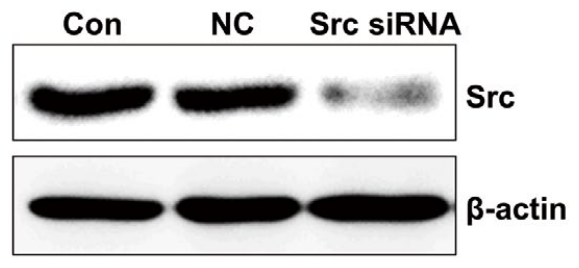

$\mathbf{E}$

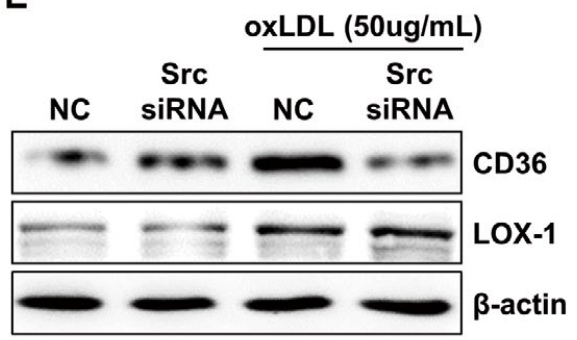

$\mathbf{F}$

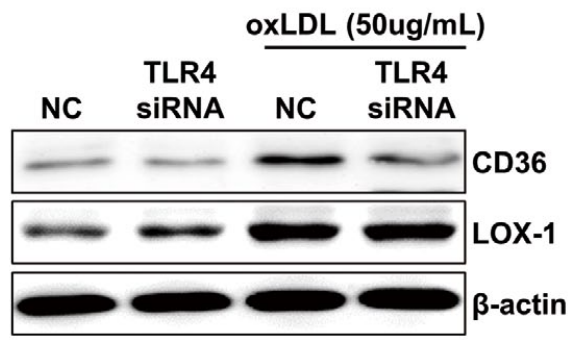

Figure 3. Toll-like receptor 4 (TLR4)-Src signaling mediates macrophage lipid accumulation. RAW264.7 macrophages were transfected with Src-specific or negative small interference RNAs (siRNA) for $48 \mathrm{~h}$. (A) Cells were then treated with oxidized lowdensity lipoprotein (oxLDL) for another $24 \mathrm{~h}$ in the presence or absence of pre-treatment with a PP2 or PP3 compound for 30 min. Oil Red O (ORO) staining was performed to exhibit intracellular lipid droplets. (B) Spectrophotometric quantifications of ORO staining was shown by measuring optical density at $500 \mathrm{~nm}$. (C) Intracellular total cholesterol levels were assessed by using a fluorometric method. (D) The efficiency of Src knockdown was analyzed by Western blotting. (E,F) RAW264.7 macrophages were transfected with Src- or TLR4-specific or negative siRNA for 48h. After incubation with oxLDL for 24h, the expression of CD36 and lectin-type oxidized LDL receptor 1 (LOX-1) had been detected by Western blotting. Data are expressed as mean \pm SEM from 3 independent tests; ${ }^{*} \mathrm{P}<0.01$ when compared to negative control (NC) transfected cells; $\# \mathrm{P}<0.01$ when compared to PP3 treatment.

\section{TLR4-Src Signaling Regulates Lipid Accumulation in Macrophages}

To investigate the role of Src in macrophage lipid accumulation, Src-specific siRNA (Figure 3) or the Src kinase inhibitor, PP2, was introduced to either reduce Src expression levels or to block its activity. PP3, an inactive PP2 analog, was also applied as a control. Intracellular lipid levels were analyzed both by ORO staining and total cholesterol quantification. We found enhanced lipid accumulation in response to oxLDL exposure was significantly reduced by Src knockdown and PP2 treatment (Figures 3A-C). Additionally, TLR4 knockdown markedly inhibited intracellular lipid accumulation in macrophages (Figure S1). Moreover, TLR4 or Src knockdown significantly attenuated the expression of CD36 but not

\section{LOX-1 (Figures 3E,F).}

oxLDL Induces TLR4 Interaction With Src in Macrophages Immunoprecipitation assays were then performed to better characterize mechanisms involved in the TLR4-Src signaling. We found that TLR4 associated with Src in RAW264.7 cells after oxLDL stimulation for 30 and $60 \mathrm{~min}$ (Figure 4A). Furthermore, co-localization of TLR4 and Src on the cell membrane upon oxLDL exposure was also detected by immunofluorescence (Figure 4B). In addition, this interaction was unaffected by TRAF6 knockdown (Figure 4C). No detectable interaction of TLR4 with Fyn and Lyn, other members in the Src Family Kinases (SFKs) group, was observed (Figure 4D). 
A
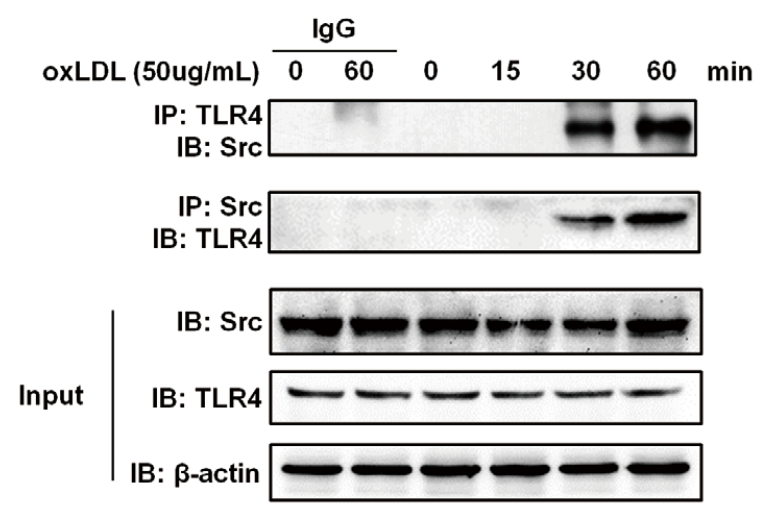

B

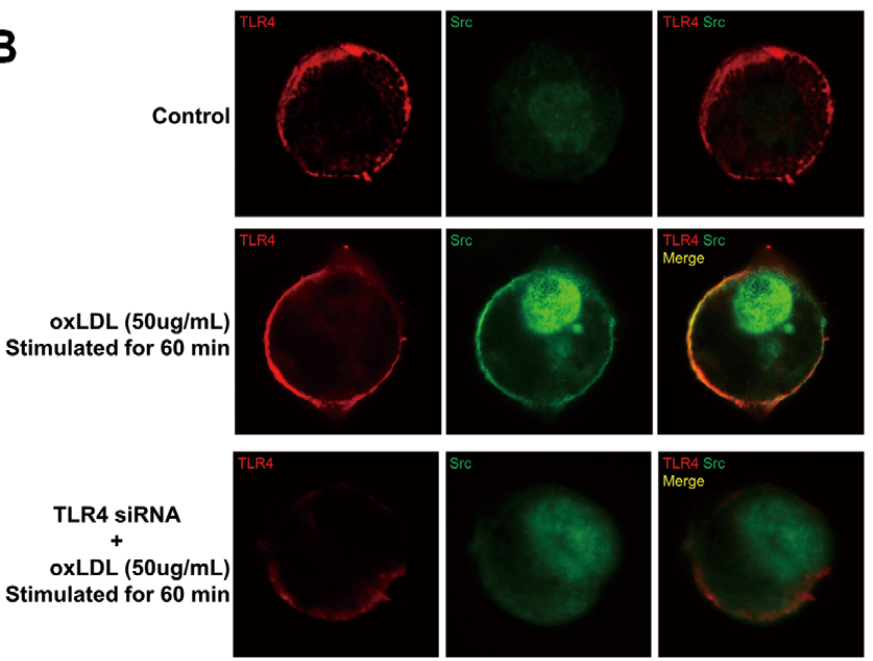

C

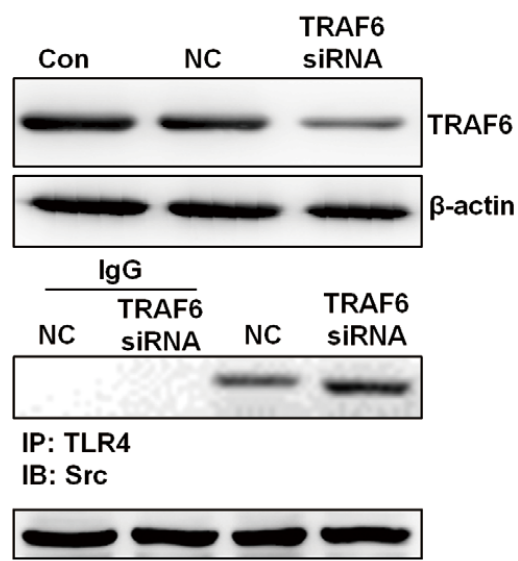

IB: Src

D
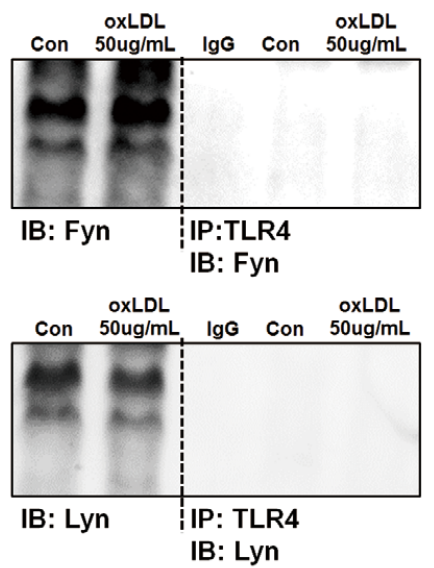

Figure 4. Toll-like receptor 4 (TLR4) interacts with Src on the cell membrane upon oxidized low-density lipoprotein (oxLDL) stimulation. (A) Immunoprecipitation assays were performed to analyze interactions between TLR4 and Src after oxLDL exposure for indicated times. (B) RAW264.7 cells treated either with negative control (NC) or TLR4-specific small interference RNAs (siRNA) were then stimulated with or without oxLDL. Subcellular localization of TLR4 and Src were assayed by immunofluorescence. A yellow color in the merge indicates co-localization. (C) RAW264.7 macrophages transfected either with TRAF6-specific or NC siRNA were stimulated with oxLDL for $60 \mathrm{~min}$. Interaction of TLR4 with Src in the presence or absence of TRAF6 was assayed by immunoprecipitation. (D) RAW264.7 cells were incubated with or without oxLDL for 60 min. Interactions of TLR4 with Fyn or Lyn were analyzed by immunoprecipitation.

\section{Discussion}

A major finding of our study is that TLR4-Src signaling plays a crucial role in macrophage lipid accumulation. We showed that inhibition of Src significantly reduced intracellular lipid contents following oxLDL exposure. Furthermore, our data suggest that TLR4 interacts with Src kinase upon oxLDL exposure on the cell membrane, thereby facilitating Src activation and lipid uptake.

In this study, we found that the inhibition of Src either by siRNA or synthetic chemicals dramatically suppressed lipid accumulation in macrophages. It is likely that Src signaling regulates macrophage lipid levels via multiple mechanisms. First, Src activation leads to cytoskeleton rearrangement in macrophages, which affects cell adhesion, ${ }^{23,24}$ spreading, ${ }^{25,26}$ and lipid uptake. ${ }^{27}$ Second, Src signaling leads to activation of c-Jun $\mathrm{N}$-terminal kinase and enhances the transactivity of
c-Jun in response to LPS, thereby promoting the expression of pro-inflammatory genes. ${ }^{28}$ Thus, Src could alter the expression of a variety of genes involved in lipid influx and efflux to affect intracellular lipid contents.

Among various ligands for TLR4, LPS from Gram-negative bacteria has been well studied. ${ }^{29-31}$ The TLR4 signaling cascade in response to LPS is dependent on recruited adaptor proteins and can be broadly divided into myeloid differentiation factor 88 (MyD88)-dependent and independent pathways, both leading to the activation of the nuclear factor $\kappa \mathrm{B}(\mathrm{NF}-\kappa \mathrm{B})$ pathway and expression of target pro-inflammatory genes. ${ }^{32-34}$ CD14 has been considered mainly as a molecule that concentrates and delivers LPS to TLR4/MD-2, thereby facilitating TLR4 activation. ${ }^{35}$ CD14 recruited Lyn, a member from the Src family kinases group, to participate in the signaling pathway of TLR $4 .^{36}$ The function of translocation associated membrane protein 1 (TRAM) is to take part in LPS-TLR4 signaling 
to regulate the MyD88-independent pathway during the innate immune response to LPS. ${ }^{37}$ However, knockdown of MyD88 or CD14 or TRAM did not have an effect on the interaction of TLR4 and Src (Figure S2). TRAF6, a critical signaling element involved in both pathways, has been shown to physically interact with Src kinase via a proline-rich $\mathrm{SH} 3$ domain in its $\mathrm{COOH}$-terminal. ${ }^{38}$ In this study, we found for the first time that TLR4 interacts with Src kinase in RAW264.7 cells by using co-immunoprecipitation. Based on previous reports and our findings, TLR4 could associate with Src directly or indirectly via TRAF6. ${ }^{38}$ However, TRAF6 is predominately localized in the cytosol, ${ }^{39}$ whereas our immunofluorescence data revealed that Src co-localizes with TLR4 on the plasma membrane upon oxLDL treatment. Moreover, the interaction of TLR4 and Src was not affected by the knockdown of TRAF6 (Figure 4C). Thus, we propose that Src may interact with the intracellular domain of TLR4 on the plasma membrane without the involvement of TRAF6. Aside from the classical proinflammatory TLR4/TRAF6 signaling, TLR4/Src may mediate macrophage lipid uptake via a TRAF6-independent pathway. Obviously, characterization of the precise molecule mechanisms to verify this hypothesis awaits further studies and will be an important future goal.

In our studies, we found that TLR4 knockdown significantly attenuated oxLDL-induced lipid accumulation (Figure S1). Interestingly, CD36, a principle membrane receptor for oxLDL, has been shown to be associated within a signaling complex containing Src, and facilitates the internalization of oxLDL in a Src-dependent manner. ${ }^{40}$ In contrast, oxLDL binds to CD36 to trigger assembly of a heterotrimeric complex composed of CD36-TLR4-TLR6; however, TLR4-TLR6 signaling is triggered by a proximal membrane event initiated by CD36 interaction with Lyn kinase. ${ }^{41}$ Similarly, we observed that oxLDL induced CD36-TLR4-TLR6 co-localization on the cell membrane, but knockdown of TLR6 did not have an effect on Src activation (Figure S3). Hence, TLR4 and CD36 may share a common mechanism to regulate intracellular lipid levels by modulating Src activity. Moreover, knockdown of Src or TLR4 weakened CD36 expression (Figures 3E,F). These results highlight the importance of crosstalk between TLR4 and CD36 caused lipid disorder via Src in macrophages.

In summary, we for the first time demonstrated that TLR4/ Src signaling is important for macrophage lipid accumulation and atherosclerotic progression. The clinical implication of this study is that deactivation of Src or dissociation of the TLR4/Src complex by synthetic chemicals could effectively attenuate the pathogenesis of atherosclerosis.

\section{Acknowledgments}

This work was supported by grants from the National Natural Science Foundation of China (30900521, 81400211 and 81200204).

\section{References}

1. Steinberg D, Lewis A. Conner Memorial Lecture: Oxidative modification of LDL and atherogenesis. Circulation 1997; 95: 1062-1071.

2. Steinberg D, Parthasarathy S, Carew TE, Khoo JC, Witztum JL. Beyond cholesterol. Modifications of low-density lipoprotein that increase its atherogenicity. N Engl J Med 1989; 320: 915-924.

3. De Paoli F, Staels B, Chinetti-Gbaguidi G. Macrophage phenotypes and their modulation in atherosclerosis. Circ J 2014; 78: 1775-1781.

4. Fukuda D, Aikawa M. Expanding role of delta-like 4 mediated notch signaling in cardiovascular and metabolic diseases. Circ J 2013; 77: $2462-2468$

5. Hansson GK. Inflammatory mechanisms in atherosclerosis. J Thromb Haemost 2009; 7(Suppl 1): 328-331.

6. Li M, Lin J, Wang Z, He S, Ma X, Li D. Oxidized low-density lipoprotein-induced proinflammatory cytokine response in macro- phages are suppressed by CD4CD25(+)Foxp3(+) regulatory T cells through downregulating toll like receptor 2-mediated activation of NF-kappaB. Cell Physiol Biochem 2010; 25: 649-656.

7. Mo ZC, Xiao J, Tang SL, Ouyang XP, He PP, Lv YC, et al. Advanced oxidation protein products exacerbates lipid accumulation and atherosclerosis through downregulation of ATP-binding cassette transporter A1 and G1 expression in apolipoprotein E knockout mice. Circ J 2014; 78: 2760-2770.

8. Kume N, Moriwaki H, Kataoka H, Minami M, Murase T, Sawamura $\mathrm{T}$, et al. Inducible expression of LOX-1, a novel receptor for oxidized LDL, in macrophages and vascular smooth muscle cells. Ann NY Acad Sci 2000; 902: 323-327.

9. Nicholson AC, Frieda S, Pearce A, Silverstein RL. Oxidized LDL binds to CD36 on human monocyte-derived macrophages and transfected cell lines: Evidence implicating the lipid moiety of the lipoprotein as the binding site. Arterioscler Thromb Vasc Biol 1995; 15: $269-275$.

10. Choi SH, Harkewicz R, Lee JH, Boullier A, Almazan F, Li AC, et al. Lipoprotein accumulation in macrophages via toll-like receptor4-dependent fluid phase uptake. Circ Res 2009; 104: 1355-1363.

11. Stoletov K, Fang L, Choi SH, Hartvigsen K, Hansen LF, Hall C, et al. Vascular lipid accumulation, lipoprotein oxidation, and macrophage lipid uptake in hypercholesterolemic zebrafish. Circ Res 2009; 104: $952-960$

12. Yang K, He YS, Wang XQ, Lu L, Chen QJ, Liu J, et al. MiR-146a inhibits oxidized low-density lipoprotein-induced lipid accumulation and inflammatory response via targeting toll-like receptor 4. FEBS Lett 2011; 585: 854-860.

13. Kefalas P, Brown TR, Brickell PM. Signalling by the p60c-src family of protein-tyrosine kinases. Int J Biochem Cell Biol 1995; 27: $551-563$.

14. Inge LJ, Fowler AJ, Paquette KM, Richer AL, Tran N, Bremner RM. Dasatinib, a small molecule inhibitor of the Src kinase, reduces the growth and activates apoptosis in pre-neoplastic Barrett's esophagus cell lines: Evidence for a noninvasive treatment of high-grade dysplasia. J Thorac Cardiovasc Surg 2013; 145: 531-538.

15. Rossi A, Schenone S, Angelucci A, Cozzi M, Caracciolo V, Pentimalli F, et al. New pyrazolo-[3,4-d]-pyrimidine derivative Src kinase inhibitors lead to cell cycle arrest and tumor growth reduction of human medulloblastoma cells. FASEB J 2010; 24: 2881-2892.

16. Mathew S, George SP, Wang Y, Siddiqui MR, Srinivasan K, Tan L, et al. Potential molecular mechanism for c-Src kinase-mediated regulation of intestinal cell migration. J Biol Chem 2008; 283: 22709-22722

17. Timpson P, Jones GE, Frame MC, Brunton VG. Coordination of cell polarization and migration by the Rho family GTPases requires Src tyrosine kinase activity. Curr Biol 2001; 11: 1836-1846.

18. Hiscox S, Jordan NJ, Morgan L, Green TP, Nicholson RI. Src kinase promotes adhesion-independent activation of FAK and enhances cellular migration in tamoxifen-resistant breast cancer cells. Clin Exp Metastasis 2007; 24: 157-167.

19. Yipp BG, Robbins SM, Resek ME, Baruch DI, Looareesuwan S, Ho M. Src-family kinase signaling modulates the adhesion of Plasmodium falciparum on human microvascular endothelium under flow. Blood 2003; 101: 2850-2857.

20. Collins RF, Touret N, Kuwata H, Tandon NN, Grinstein S, Trimble WS. Uptake of oxidized low density lipoprotein by CD36 occurs by an actin-dependent pathway distinct from macropinocytosis. $J$ Biol Chem 2009; 284: 30288-30297.

21. Frontini MJ, O'Neil C, Sawyez C, Chan BM, Huff MW, Pickering JG. Lipid incorporation inhibits Src-dependent assembly of fibronectin and type I collagen by vascular smooth muscle cells. Circ Res 2009; 104: 832-841.

22. Kritharides L, Jessup W, Mander EL, Dean RT. Apolipoprotein A-Imediated efflux of sterols from oxidized LDL-loaded macrophages. Arterioscler Thromb Vasc Biol 1995; 15: 276-289.

23. Chang MY, Huang DY, Ho FM, Huang KC, Lin WW. PKC-dependent human monocyte adhesion requires AMPK and Syk activation. PLoS One 2012; 7: e40999, doi:10.1371/journal.pone.0040999.

24. Davidson L, Pawson AJ, Millar RP, Maudsley S. Cytoskeletal reorganization dependence of signaling by the gonadotropin-releasing hormone receptor. J Biol Chem 2004; 279: 1980-1993.

25. Ishida T, Ishida M, Suero J, Takahashi M, Berk BC. Agonist-stimulated cytoskeletal reorganization and signal transduction at focal adhesions in vascular smooth muscle cells require c-Src. J Clin Invest 1999; 103: 789-797.

26. Wang PS, Chou FS, Porchia L, Saji M, Pinzone JJ. Troglitazone inhibits cell migration, adhesion, and spreading by modulating cytoskeletal rearrangement in human breast cancer cells. Mol Carcinog 2008; 47: 905-915. 
27. Kasahara K, Nakayama Y, Sato I, Ikeda K, Hoshino M, Endo T, et al. Role of Src-family kinases in formation and trafficking of macropinosomes. J Cell Physiol 2007; 211: 220-232.

28. Smolinska MJ, Horwood NJ, Page TH, Smallie T, Foxwell BM Chemical inhibition of Src family kinases affects major LPS-activated pathways in primary human macrophages. Mol Immunol 2008; 45: $990-1000$.

29. Hoshino K, Takeuchi O, Kawai T, Sanjo H, Ogawa T, Takeda Y, et al. Cutting edge: Toll-like receptor 4 (TLR4)-deficient mice are hyporesponsive to lipopolysaccharide: Evidence for TLR4 as the Lps gene product. J Immunol 1999; 162: 3749-3752.

30. Nagai Y, Akashi S, Nagafuku M, Ogata M, Iwakura Y, Akira S, et al. Essential role of MD-2 in LPS responsiveness and TLR4 distribution. Nat Immunol 2002; 3: 667-672.

31. Poltorak A, He X, Smirnova I, Liu MY, Van Huffel C, Du X, et al. Defective LPS signaling in $\mathrm{C} 3 \mathrm{H} / \mathrm{HeJ}$ and $\mathrm{C} 57 \mathrm{BL} / 10 \mathrm{ScCr}$ mice: Mutations in Tlr4 gene. Science 1998; 282: 2085-2088.

32. Cronin JG, Turner ML, Goetze L, Bryant CE, Sheldon IM. Toll-like receptor 4 and MYD88-dependent signaling mechanisms of the innate immune system are essential for the response to lipopolysaccharide by epithelial and stromal cells of the bovine endometrium. Biol Reprod 2012; 86: 51.

33. Mandal P, Pratt BT, Barnes M, McMullen MR, Nagy LE. Molecular mechanism for adiponectin-dependent M2 macrophage polarization: Link between the metabolic and innate immune activity of fulllength adiponectin. J Biol Chem 2011; 286: 13460-13469.

34. Qi HY, Shelhamer JH. Toll-like receptor 4 signaling regulates cytosolic phospholipase A2 activation and lipid generation in lipopolysaccharide-stimulated macrophages. J Biol Chem 2005; 280: 38969-38975.

35. da Silva Correia J, Soldau K, Christen U, Tobias PS, Ulevitch RJ. Lipopolysaccharide is in close proximity to each of the proteins in its membrane receptor complex: Transfer from CD14 to TLR4 and MD-2. J Biol Chem 2001; 276: 21129-21135.

36. Stefanova I, Corcoran ML, Horak EM, Wahl LM, Bolen JB, Horak ID. Lipopolysaccharide induces activation of CD14-associated pro- tein tyrosine kinase p53/56lyn. J Biol Chem 1993; 268: $20725-$ 20728.

37. Palsson-McDermott EM, Doyle SL, McGettrick AF, Hardy M, Husebye $\mathrm{H}$, Banahan $\mathrm{K}$, et al. TAG, a splice variant of the adaptor TRAM, negatively regulates the adaptor MyD88-independent TLR4 pathway. Nat Immunol 2009; 10: 579-586.

38. Liu A, Gong P, Hyun SW, Wang KZ, Cates EA, Perkins D, et al. TRAF6 protein couples Toll-like receptor 4 signaling to Src family kinase activation and opening of paracellular pathway in human lung microvascular endothelia. J Biol Chem 2012; 287: 16132-16145.

39. Verstak B, Nagpal K, Bottomley SP, Golenbock DT, Hertzog PJ, Mansell A. MyD88 adapter-like (Mal)/TIRAP interaction with TRAF6 is critical for TLR2- and TLR4-mediated NF-kappaB proinflammatory responses. J Biol Chem 2009; 284: 24192-24203.

40. Chen K, Febbraio M, Li W, Silverstein RL. A specific CD36-dependent signaling pathway is required for platelet activation by oxidized low-density lipoprotein. Circ Res 2008; 102: 1512-1519.

41. Stewart CR, Stuart LM, Wilkinson K, van Gils JM, Deng J, Halle A, et al. CD36 ligands promote sterile inflammation through assembly of a Toll-like receptor 4 and 6 heterodimer. Nat Immunol 2010; 11: $155-161$.

\section{Supplementary Files}

Supplementary File 1

Figure S1. (A) Boiled oxidized low-density lipoprotein (oxLDL) no longer promotes Src phosphorylation.

Figure S2. (A) The efficiency of myeloid differentiation factor 88 (MyD88) or translocation associated membrane protein 1 (TRAM) or CD14 knockdown was analyzed by Western blotting.

Figure S3. (A) RAW264.7 cells treated with oxidized low-density lipoprotein (oxLDL) for $60 \mathrm{~min}$.

Please find supplementary file(s); http://dx.doi.org/10.1253/circj.CJ-15-0345 